\title{
ON THE GROUP STRUCTURE IN HOMOTOPY GROUPS
}

\author{
MASATAKE KURANISHI
}

\section{Introtuction}

Usually the group structure in a homotopy group is defined directly and explicitly. But the algebraic approach to the topology, now common, seems to raise the following question: is that the only group sturcture which is natural from the algebraic topological point of view? On the other hand, several algebraists have begun to feel a necessity to construct a "homotopy or cohomotopy theory of groups," and it may be allowed to say that one of the first steps to the problem is the axiomatization of homotopy groups. Our first question is of course a special case of the latter problem.

In this note, we propose to show that the usual group sturcture in homotopy groups is indeed the only natural one; the strict definition of naturality will be given below. But for the sake of convenience, we formulate our theorem in more general form, that is, in a form of axioms (of homotopy groups).

In our system of axioms for homotopy theory, we introduce path spaces and zero-dimensional homotopy classes. But these circumstances seem to suggest that our axioms are not yet in a final form of the axioms of homotopy groups.

The writer is greatly indebted to Prof. Nakayama for his suggestion to the problem and Dr. Shimada for many useful remarks and discussions. The writer wishes also to express his gratitude to Prof. Chevalley for the suggestion of the generalized form of a lemma in this paper.

\section{§. Preliminaries and the statement of the theorem}

(1.1) A set $G$ is called a set with a neutral element, if there is a specific element in $G$ which is called the neutral element of $G$. We shall denote generally by $e$ the neutral element. Any group is also considered naturally as a set with the neutral element. Let $G$ and $H$ be two sets with the neutral elements.

Received April 15, 1954. 
A homomorphism $f$ of $G$ into $H$.

$$
f: G \rightarrow H,
$$

is a single valued function from $G$ to $H$ such that the neutral element of $G$ is mapped to that of $H$. A homomorphism $f: G \rightarrow H$ is called an isomorphism if the function $f$ is one-to-one.

Let $A$ be a topological space and let $a$ be a point of $A$. Defining the neutral element to be the arc-wise component of $A$ containing $a$, the set of totality of arc-wise components of $A$ forms a set with the neutral element, which is denoted by $\pi_{0}(A, a)$. For any continuous mapping $f:(A, a) \rightarrow(B, b)$, we can naturally associate the homomorphism $f_{\pi}: \pi_{0}(A, a) \rightarrow \pi_{0}(B, b)$, namely $f_{\pi}$ (the component containing $\left.a^{\prime}\right)=$ the component containing $f\left(a^{\prime}\right)$. By generalizing the usual definition of the set $\pi_{n}(A, B, b)$ to the case $n=1$, we obtain also the set with the neutral element $\pi_{1}(A, B, b)$.

(1.2) Let $G_{n}(n=1,2, \ldots)$ be sets with neutral elements, and $f_{n}(n=1$, $2, \ldots$ ) be homomorphism of $G_{n+1}$ to $G_{n}$. Then they are called exact sequence if the following conditions are satisfied:

(1) the image of $f_{m+1}=$ the complete inverse image of $e$ by $f_{m}$ for any $m \gtrsim 1$.

(2) if both $G_{m}$ and $G_{m+3}$ consist of only one element, then $f_{m+2}$ is an isomorphism for any $m \geqslant 1$.

In the case that both $G_{m+1}$ and $G_{m+2}$ are groups and $f_{m+2}$ is a homomorphism of group structures, the condition (2) follows from (1).

(1.3) Suppose $G$ and $H$ are groups or sets with the neutral elements. In the sequel, we shall say that a mapping $f: G \rightarrow H$ is a homomorphism, in the following two cases:

(1) both $G$ and $H$ are groups, and $f$ is a homomorphism of group structures.

(2) At least one of $G$ and $H$ is not a group, and $f$ is a homomorphism considering both $G$ and $H$ as sets with the neutral elements.

We shall make the same abbreviation for "isomorphisms."

(1.4) Let $A$ be a topological space, and $B$ and $C$ be subspaces of $A$. Introducing the so-called compact-open topology in the set of totality of paths in $A$ starting from points of $B$ and terminating in points of $C$, we obtain a topological space, which is denoted by $\Omega_{B, C}(A)$. If $A^{\prime} \cong A, B^{\prime} \cong B$, and $C^{\prime} \cong C$, then 
$\Omega_{B^{\prime}, c^{\prime}}\left(A^{\prime}\right)$ can be considered to be a subspace of $\Omega_{B, C}(A)$.

(1.5) Let $A$ be a topological space. Mapping the curve $c \in \Omega_{b, A}(A)$ to the end point of $c$, we obtain a continuous mapping $\tilde{\rho}$, which defines an "espace fibré" ( $\left.\Omega_{b, A}(A), A, \widetilde{\rho}\right)$. Since $\tilde{\rho}^{-1}(B)=\Omega_{b, B}(A) \cong \Omega_{b, A}(A)$, it is well known that the mapping $\rho:\left(\Omega_{b, A}(A), \Omega_{b, B}(A), \Omega_{b, b}(b)\right) \rightarrow(A, B, b)$ determined by $\tilde{\rho}$ induces an isomorphism $\rho_{\pi}: \pi_{n}\left(\Omega_{b, A}(A), \Omega_{b, B}(A), \Omega_{b, b}(b)\right) \rightarrow \pi_{n}(A, B, b)$.

(1.6) A homotopy theory $(I)$ is defined to be following four functions satisfying the eight axioms listed below.

(1) For each pair $(A, a)$, where $a$ is a point of $A$, and for each integer $n \geqslant 1$, there is associated a group $\Pi_{n}(A, a)$, and also is associated a set with neutral element $\Pi_{0}(A, a)$. For each triple $(A, B, b)$, where $b \in B \subseteq A$, and for each integer $n \geqslant 2$, there is associated a group $\Pi_{n}(A, B, b)$, and also is associated a set with neutral element $\Pi_{1}(A, B, b)$.

(2) For each $(A, B, b)$, there is defined a homomorphism $\partial_{\mathrm{n}}: \Pi_{n+1}(A, B, b)$ $\rightarrow \Pi_{n}(B, b),(n \geqslant 0)$. The homomorphism $\partial_{\mathrm{n}}$ is called a boundary homomorphism.

(3) For each $(A, B, b)$, there is defined a homomorphism $j_{\mathrm{n}}: \Pi_{n}(A, b)$ $\rightarrow \Pi_{n}(A, B, b),(n \geqslant 1)$. The homomorphism $j_{\mathrm{n}}$ is called a relativisation homomorphism.

(4) For each continuous mapping $f:(A, B, b) \rightarrow(P, Q, q)(f:(A, a)$ $\rightarrow(P, p))$, there is defined a homomorphism $f_{n}: \Pi_{n}(A, B, b) \rightarrow \Pi_{n}(P, Q, q)$, $(n \geqslant 1),\left(f_{\mathrm{n}}: \Pi_{n}(A, a) \rightarrow \Pi_{n}(P, p), n \geqslant 0\right) . \quad f_{\Pi}$ is called the homomorphism induced by $f$.

They satisfy the following axioms;

(A.1) if $f:(A, a) \rightarrow(A, a)$ is the identity mapping, $f_{\mathrm{n}}$ is also the identity mapping,

$(A .2) \quad(f \circ g)_{\mathrm{n}}=f_{\mathrm{n}} \circ g_{\mathrm{n}}$,

(A.3) if $f \simeq g$, then $f_{\mathrm{n}}=g_{\mathrm{n}}$,

$(A .4) \longrightarrow \Pi_{n}(B, b) \stackrel{i_{\mathrm{n}}}{\longrightarrow} \Pi_{n}(A, b) \stackrel{j_{\mathrm{n}}}{\longrightarrow} \Pi_{n}(A, B, b) \stackrel{\partial_{\mathrm{n}}}{\longrightarrow} \Pi_{n-1}(B, b) \longrightarrow$ $\ldots \rightarrow \Pi_{1}(A, B, b) \stackrel{\partial \mathrm{n}}{\longrightarrow} \Pi_{0}(B, b) \stackrel{j_{\mathrm{n}}}{\longrightarrow} \Pi_{0}(A, b)$

where $i$ is the inclusion mapping, is an exact sequence in the sense defined in (1.2).

(A.5) for any $f:(A, B, b) \rightarrow(P, Q, q)$, the following diagrams are commutative: 


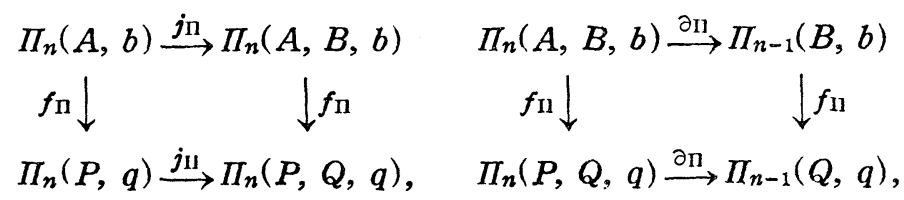

(A.6) for each $(A, a)$

$$
i_{\Pi}: \Pi_{n}\left(\Omega_{a, A}(A), \Omega_{a, a}(A) ; \Omega_{a, a}(a)\right) \rightarrow \Pi_{n}\left(\Omega_{A, A}(A), \Omega_{A, a}(A), \Omega_{a, a}(a)\right)
$$

where $i$ is the inclusion mapping, is an isomorphism for each integer $n \geqslant 1$,

(A.7) let $a$ be a space consisting of one point, then $\Pi_{n}(a, a)=\{e\}$ for each integer $n \geqslant 1$,

(A.8) $\Pi_{0}(A, a)=\pi_{0}(A, a)$, and $f_{\mathrm{n}}: \Pi_{0}(A, a) \rightarrow \Pi_{0}(P, p)$ is just the homomorphism $f_{\pi}: \pi_{0}(A, a) \rightarrow \pi_{0}(P, p)$ defined in (1.1).

(1.7) Let $(I I)$ and $\left(\Pi^{\prime}\right)$ be two homotopy theories. Then $(I I)$ and $\left(\Pi^{\prime}\right)$ are called equivalent to each other, if there exist one-to-one mappings $\omega_{n, n}$, : $\Pi_{n}(A, B, b) \rightarrow \Pi_{n}^{\prime}(A, B, b), \Pi_{n}(A, a) \rightarrow \Pi_{n}^{\prime}(A, a)$ satisfying the following conditions :

(1) $\omega_{\mathrm{n}, \mathrm{n},}$ 's are isomorphisms for every $(A, B, b)$ and $(A, a)$, or $\omega_{\mathrm{n}, \mathrm{n}}$ 's are inverse-isomorphisms for every $(A, B, b)$ and $(A, a)$,

(2) the following diagrams are commutative:

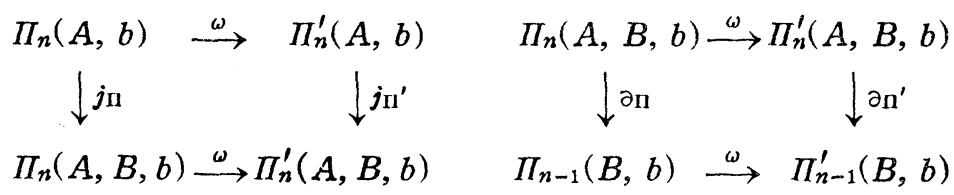

(3) for each mappings $f:(A, B, b) \rightarrow(P, Q, q)$, and $g:(A, a) \rightarrow(P, p)$, the following diagrams are commutative :

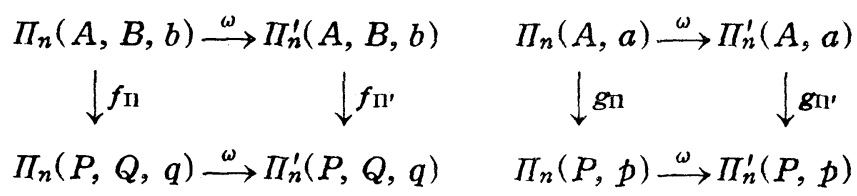

(1.8) THEOREM: Any homotopy theory is equivalent to the usual homotopy theory.

\section{§ 2. Immediate corollaries from the axioms}

(2.1) Let $X$ be a space and let $x$ be a point of $X$. Then $\Pi_{n}(X, X, x)=\{e\}$ for $n \gtrsim 1$. 
(2.2) $j_{\mathrm{n}}: \Pi_{n}(X, x) \rightarrow \Pi_{n}(X, x, x)$ is an isomorphism for $n \geq 1$.

This follows from $(A .7)$ and $(A .4)$ putting $A=X, B=b=x$.

(2.3) For any $(X, Y, y), j_{\mathrm{n}}: \Pi_{n}(X, y) \rightarrow \Pi_{n}(X, Y, y)$ is equal to the composition of $j_{\mathrm{Il}}: \Pi_{n}(X, y) \rightarrow \Pi_{n}(X, y, y)$ and $i_{\mathrm{n}}: \Pi_{n}(X, y, y) \rightarrow \Pi_{n}(X, Y, y)$, where $i$ is the inclusion mapping.

This follows from the commutativity of the diagram

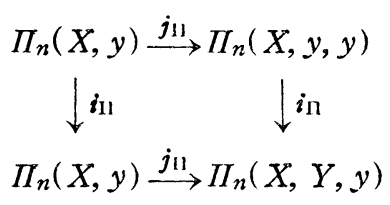

(by $(A .5))$ and $(A .1)$.

(2.4) Let $h:(X, x) \rightarrow(X, x)$ be the constant mapping, namely, $h\left(x^{\prime}\right)=x$ for every $x^{\prime} \in X$. Then the image of $h_{\mathrm{n}}=\{e\}$.

Let $h^{\prime}:(X, x) \rightarrow(x, x)$ be the mapping such that $h^{\prime}\left(x^{\prime}\right)=x$ for every $x^{\prime}$ $\in X$, and $i:(x, x) \rightarrow(X, x)$ be the inclusion mapping. Then $h=i \circ h^{\prime}$. By $(A .2), h_{\mathrm{n}}=i_{\mathrm{n}} \circ h_{11}^{\prime}$. Hence image $h_{\mathrm{n}} \leqq$ image $i_{\mathrm{n}}=$ the neutral element by (A.7).

(2.5) If $(X, x)$ is contractible, that is, if the identity mapping is homotopic to the constant mapping, then $\Pi_{n}(X, x)=\{e\}$ for $n \gtrsim 0$.

This follows from $(A .3)$ and (2.4).

(2.6) If $i:(A, B, b) \rightarrow(A, B, b)$ is the identity mapping then $i_{\mathrm{n}}$ : $\Pi_{n}(A, B, b) \rightarrow \Pi_{n}(A, B, b)$ is the identity mapping for each $n \geqslant 1$.

This comes from $(A .1),(A .4),(A .7)$, and from the so-called five lemma.

(2.7) Let $\left(A^{\prime}, B^{\prime}, b^{\prime}\right) \cong(A, B, b)$. Suppose there exists a homotopy $h_{t}$ : $(A, B, b) \rightarrow(A, B, b), \quad(0 \leqq t \leqq 1)$, such that $h_{0}=$ the identity mapping, $h_{1}(A)$ $\leqq A^{\prime}, h_{1}(B) \leqq B^{\prime}$, and such that $h_{t}\left(a^{\prime}\right)=a^{\prime}$ for $a^{\prime} \in A^{\prime}, 0 \leqq t \leqq 1$. Then $i_{\Pi}$ : $\Pi_{n}\left(A^{\prime}, B^{\prime}, b\right) \rightarrow \Pi_{n}(A, B, b)$ is isomorphism for $n \geq 1$.

Let $h^{\prime}:(A, B, b) \rightarrow\left(A^{\prime}, B^{\prime}, b\right)$ be the mapping such that $h^{\prime}(a)=h_{1}(a)$ for $a \in A$. Then clearly $h_{1}=i \circ h^{\prime}$ and $h^{\prime} \circ i=$ the identity mapping, where $i$ is the inclusion mapping $\left(A^{\prime}, B^{\prime}, b\right) \rightarrow(A, B, b)$. Hence

$$
i_{\mathrm{n}} \circ h_{\mathrm{\Pi}}^{\prime}=h_{1 \mathrm{n}}=h_{0 \mathrm{n}}=\text { the identity mapping, }
$$

and

$$
h_{11}^{\prime} \circ i_{11}=\text { the identity mapping. }
$$

(2.8) Mapping the curve $c \in \Omega_{x, X}(X)$ to the end point of $c$, we obtain the 
continuous mapping $\rho:\left(\Omega_{x, X}(X), \Omega_{x, x}(X), \Omega_{x, x}(x)\right) \rightarrow(X, x, x)$. Then $\rho_{\mathrm{M}}$ is an isomorphism for $n \geqslant 1$. This proposition is a special case of (1.5) for (II)-theory.

Let $k: X \rightarrow \Omega_{X, X}(X)$ be the mapping such that $k(y)=\Omega_{y, y}(y)$, and let $\bar{X}$ be the image of $k$. Then $k$ induces a homeomorphism between $X$ and $\bar{X}$, and so we may identify $X$ and $\bar{X}$.

For any curve $c$ in $X$ and real number $t(0 \leqq t \leqq 1)$, let $c_{t}$ be the curve in $X$ such that $c_{t}(u)=c((1-t) u)$ for $0 \leqq u \leqq 1$. Let $r_{t}:\left(\Omega_{X, X}(X), \Omega_{x, X}(X)\right.$, $\left.\Omega_{x, x}(x)\right) \rightarrow\left(\Omega_{X, X}(X), \Omega_{x, X}(X), \Omega_{x, x}(x)\right)$ be the mapping such that $r_{t}(c)=c_{t}$ for $0 \leqq t \leqq 1$. If we put $\Omega_{X, X}(X)=A, \Omega_{x, X}(X)=B, X=\bar{X}=A^{\prime}$, and $\Omega_{x, x}(x)=B=b$, $r_{t}$ satisfy the conditions of $h_{t}$ in (2.7). Hence the inclusion mapping $i_{1}:(X, x, x)$ $\rightarrow\left(\Omega_{X, X}(X), \Omega_{x, X}(X), \Omega_{x, x}(x)\right)$ induces an isomorphism $i_{1 \mathrm{n}}$.

Now we have the following commutative diagram:

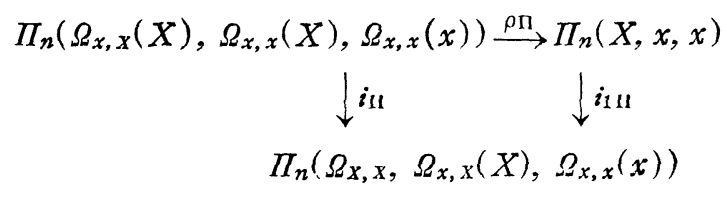

where $i$ is the inclusion mapping. (A.6) implies that $i_{\mathrm{n}}$ is an isomorphism, and, as we have just shown, $i_{1 \mathrm{n}}$ is also an isomorphism. Hence $\rho_{\mathrm{n}}$ is an isomorphism.

(2.9) We insert here an algebraic lemma which will be used in the proof of our theorem.

Lemma: Let $F(X, Y)$ be a word in two variables $X$ and $Y$. Let $F_{2}$ be a free group generated by two elements. We shall introduce in $F_{2}$ a new multiplication $\circ$, by

$$
\alpha \circ \beta=F(\alpha, \beta), \quad\left(\alpha, \beta \in F_{2}\right) .
$$

If the multiplication $\circ$ defines a group structure in the set $F_{2}$, then $F(X, Y)$ $=X Y$ or $Y X$.

The writer proved the lemma in a more restrictive case. The generalized form, listed here, is dued to Prof. Chevalley, and this generalization shortened the proof of our theorem. Independently, Messrs. Iwahori, Hattori, and Morimoto obtained elementary proofs and generalizations of our lemma. The writer wants to omit his proof, since it depends on a rather deep theorem on Lie groups and the elementary proofs will be published in this issue. 


\section{§. Canonical mapping $\omega$}

(3.1) We intend to define the canonical mapping $\omega_{n}: \pi_{n}(X, Y, y) \rightarrow$ $\Pi_{n}(X, Y, y), \pi_{n}(X, x) \rightarrow \Pi_{n}(X, x)$ by induction with respect to $n$. According to $(A .8), \pi_{0}(X, x)=\Pi_{0}(X, x)$. By definition, the identity mapping $\omega_{0}: \pi_{0}(X, x) \rightarrow$ $\Pi_{0}(X, x)$ is the canonical mapping. Now suppose that $\omega_{n-1}: \pi_{n-1}(X, x) \rightarrow \Pi_{n-1}(X, x)$ is defined for each $(X, x),(n \geqslant 1)$.

In the diagram

$$
\begin{aligned}
& \pi_{n-1}\left(\Omega_{y, Y}(X), \bar{y}\right) \stackrel{\partial_{\pi}}{\longleftarrow} \pi_{n}\left(\Omega_{y, X}(X), \Omega_{y, Y}(X), \bar{y}\right) \stackrel{\rho_{\pi}}{\longrightarrow} \pi_{n}(X, Y, y) \\
& \quad \downarrow_{\omega_{n-1}} \\
& \Pi_{n-1}\left(\Omega_{y, Y}(X), \bar{y}\right) \stackrel{\partial_{\mathrm{n}}}{\longleftarrow} \Pi_{n}\left(\Omega_{y, X}(X), \Omega_{y, Y}(X), \bar{y}\right) \stackrel{\rho_{\Pi}}{\longrightarrow} \Pi_{n}(X, Y, y),
\end{aligned}
$$

(for the definition of $\rho$, see $(2.8)$ ), where $\bar{y}=\Omega_{y, y}(y), \rho_{\pi}$ is an isomorphism by (1.5), and $\partial_{n}$ is also an isomorphism, since $\Omega_{y, X}(X)$ is contractible. By definition, $\omega_{n}=\rho_{\mathrm{n}} \circ \partial_{\Pi}^{-1} \circ \omega_{n-1} \circ \partial_{\pi} \circ \rho_{\pi}^{-1}: \pi_{n}(X, Y, y) \rightarrow \Pi_{n}(X, Y, y)$ is the canonical mapping for each $(X, Y, y)$. We define the canonical mapping $\omega_{n}: \pi_{n}(X, x)$ $\rightarrow \Pi_{n}(X, x)$ to be the composition of mappings

$$
\pi_{n}(X, x) \stackrel{j_{\pi}}{\longrightarrow} \pi_{n}(X, x, x) \stackrel{\omega_{n}}{\longrightarrow} \Pi_{n}(X, x, x) \stackrel{j_{\Pi}^{-1}}{\longrightarrow} \Pi_{n}(X, x),
$$

where $j_{\pi}$ and $j_{\mathrm{n}}$ are the relativisation isomorphisms (cf. (2.2)).

(3.2) The canonical mapping $\omega_{n}: \pi_{n}(X, x) \rightarrow \Pi_{n}(X, x)$ is one-to-one for each $(X, x)$ and $n \geqslant 0$.

$\omega_{0}: \pi_{0}(X, x) \rightarrow \Pi_{0}(X, x)$ is an isomorphism by definition. Suppose that $\omega_{n-1}: \pi_{n-1}(X, x) \rightarrow \Pi_{n-1}(X, x)$ is one-to-one. Then in the definition of $\omega_{n}$ : $\pi_{n}(X, x) \rightarrow \Pi_{n}(X, x)$, the mappings $j_{\pi}, \rho_{\pi}^{-1}, \partial_{\pi}, \omega_{n-1}, \partial_{\Pi}^{-1}, \rho_{\mathrm{n}}$, and $j_{\Pi}^{-1}$ are isomorphisms (cf. (31) and (2.8)), and so $\omega_{n}$ is one-to-one.

(3.3) But at this stage we don't know if they are isomorphisms of group structures (when they are mappings between groups). If we find that $\omega_{1}$ : $\pi_{1}(X, x) \rightarrow \Pi_{1}(X, x)$ is a homomorphism of group structures for any $(X, x)$, then it follows automatically that $\omega_{n}: \pi_{n}(X, x) \rightarrow \Pi_{n}(X, x)$ is an isomorphism for any $(X, x)$ and $n \geqslant 0$, by the same arguments as in (3.2).

(3.4) For any continuous mappings $f:(X, Y, y) \rightarrow(X, Y, y)$ and $f:(X, x)$ $\rightarrow\left(X^{\prime}, x^{\prime}\right)$, the diagrams 
(1) $n$

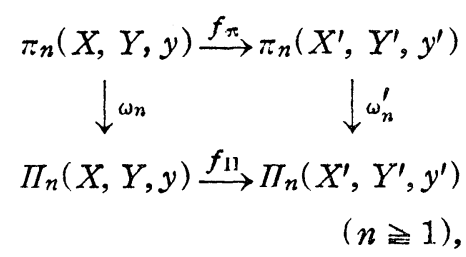

$(2)_{n}$

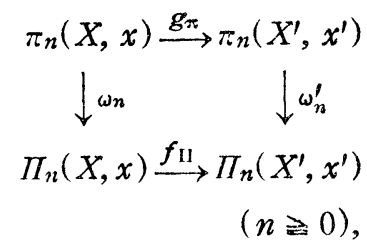

are commutative.

If $n=0$, the diagram $(2)_{0}$ is clearly commutative by $(A .8)$. We prove the proposition by the following inductions:

a) if $(2)_{n-1}$ is true, then $(1)_{n}$ is true,

$b)$ if $(1)_{n}$ is true, then $(2)_{n}$ is true.

\section{Proof of $a$ )}

Consider the following diagram:

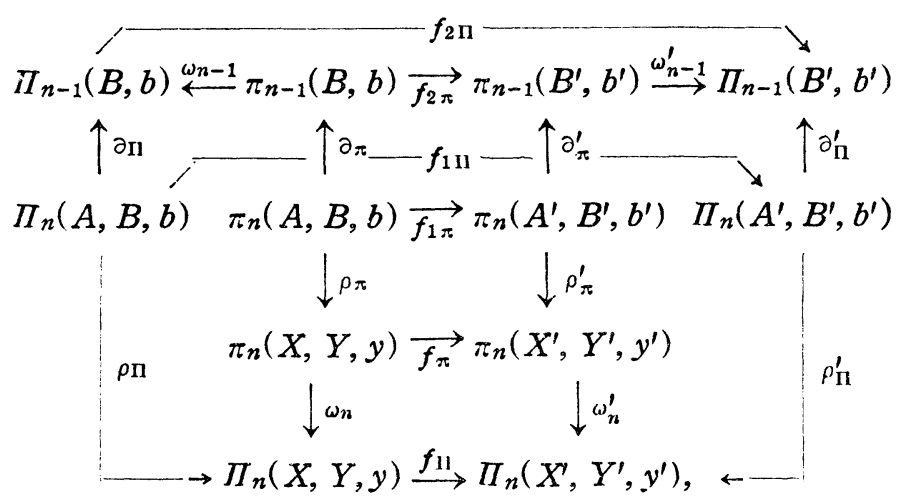

where $A=\Omega_{y, X}(X), A^{\prime}=\Omega_{y^{\prime}, X^{\prime}}\left(X^{\prime}\right)$,

$B=\Omega_{y, Y}(X), B^{\prime}=\Omega_{y^{\prime}, Y^{\prime}}\left(X^{\prime}\right), \quad b=\Omega_{y, y}(y), b^{\prime}=\Omega_{y^{\prime}, y^{\prime}}\left(y^{\prime}\right)$,

$\rho$ and $\rho^{\prime}$ are mappings defined in (2.8), and $f_{1}$ and $f_{2}$ are

mapping naturally induced by $f$.

Then the assumption of our induction and the axioms of homotopy theories imply that the diagram is commutative. Hence

$$
\begin{aligned}
\omega_{n}^{\prime} \circ f_{\pi} & =\rho_{n}^{\prime} \circ \partial_{1}^{\prime-1} \circ \omega_{n-1}^{\prime} \circ \partial_{\pi}^{\prime} \circ \rho_{\pi}^{\prime-1} \circ f_{\pi} \circ \rho_{\pi} \circ \rho_{\pi}^{-1} \\
& =\rho_{\pi}^{\prime} \circ \partial_{n}^{-1} \circ \omega_{n-1}^{\prime} \circ \partial_{\pi}^{\prime} \circ \rho_{\pi}^{\prime-1} \circ \rho_{\pi}^{\prime} \circ f_{1 \pi} \circ \rho_{\pi}^{-1} \\
& =\rho_{n}^{\prime} \circ \partial_{n}^{\prime-1} \circ \omega_{n-1}^{\prime} \circ \partial_{\pi}^{\prime} \circ f_{1 \pi} \circ \rho_{\pi}^{-1} \\
& =\rho_{n}^{\prime} \circ \partial_{n}^{\prime-1} \circ \omega_{n-1}^{\prime} \circ f_{2 \pi} \circ \partial_{\pi} \circ \rho_{\pi}^{-1} \\
& =\rho_{n}^{\prime} \circ \partial_{n}^{\prime-1} \circ f_{2 n} \circ \omega_{n-1} \circ \partial_{\pi} \circ \rho_{\pi}^{-1} \\
& =\rho_{n}^{\prime} \circ f_{1 n} \circ \partial_{n}^{-1} \circ \omega_{n-1} \circ \partial_{\pi} \circ \rho_{\pi}^{-1}
\end{aligned}
$$




$$
\begin{aligned}
& =f_{\mathrm{I}} \circ \rho_{\mathrm{n}} \circ \partial_{\mathrm{I}}^{-1} \circ \omega_{n-1} \circ \partial_{\pi} \circ \rho_{\pi}^{-1} \\
& =f_{\mathrm{II}} \circ \omega_{n},
\end{aligned}
$$

Proof of $b$ )

Consider the diagram:

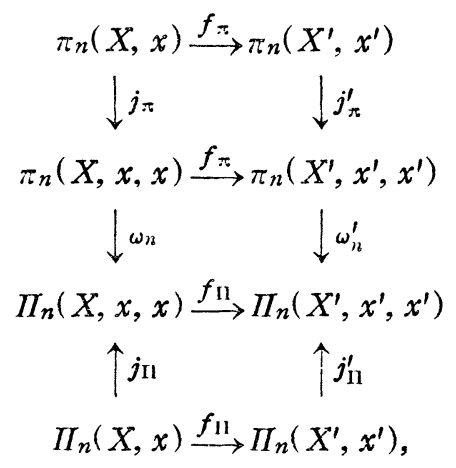

then the definition of the canonical mapping $\omega_{n}: \pi_{n}(X, x) \rightarrow \Pi_{n}(X, x)$ and $(a)$ show that the almost part of the diagram is commutative. Therefore using this diagram we can easily deduce the commutativity of the diagram $(2)_{n}$.

(3.5) For any $(X, Y, x)$, the diagram

$$
\begin{gathered}
\pi_{n}(X, Y, y) \stackrel{\omega_{n}}{\longrightarrow} \Pi_{n}(X, Y, y) \\
\downarrow \partial_{\pi} \quad \downarrow \partial_{11} \\
\pi_{n-1}(Y, y) \stackrel{\omega_{n-1}}{\longrightarrow} \Pi_{n-1}(Y, y)
\end{gathered}
$$

is commutative for $n \geqslant 1$.

To show (3.5), consider the diagram

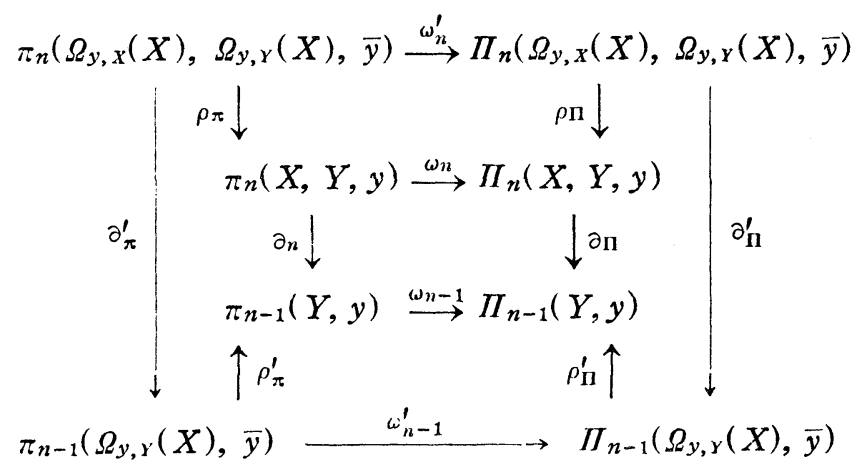

where $\bar{y}=\Omega_{y, y}(y)$. Then 


$$
\begin{aligned}
\omega_{n-1} \circ \partial_{\pi} & =\omega_{n-1} \circ \partial_{\pi} \circ \rho_{\pi} \circ \rho_{\pi}^{-1} \\
& =\omega_{n-1} \circ \rho_{\pi}^{\prime} \circ \partial_{\pi}^{\prime} \circ \rho_{\pi}^{-1} \\
& =\rho_{\Pi 1}^{\prime} \circ \omega_{n-1}^{\prime} \circ \partial_{\pi}^{\prime} \circ \rho_{\pi}^{-1} \\
& =\partial_{\mathrm{n}} \circ \rho_{\Pi} \circ \partial_{\pi}^{\prime-1} \circ \omega_{n-1}^{\prime} \circ \partial_{\pi}^{\prime} \circ \rho_{\pi}^{-1} \\
& =\partial_{11} \circ \omega_{n} .
\end{aligned}
$$

(3.6) The diagram

$$
\begin{array}{cc}
\pi_{n}(X, y) \stackrel{\omega_{n}}{\longrightarrow} \Pi_{n}(X, y) \\
\downarrow_{\pi} \stackrel{j_{\pi}}{j_{n}} \\
\pi_{n}(X, Y, y) \stackrel{\omega_{n}}{\longrightarrow} \Pi_{n}(X, Y, y)
\end{array}
$$

is commutative for any $(X, Y, y)$ and $n \geqslant 1$.

By definition, the diagram

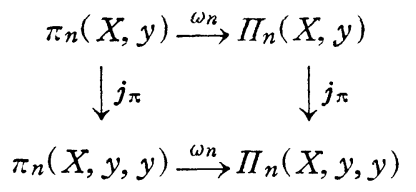

is commutative. Hence (3.6) follows from (2.3) and (3.4).

\section{$\S 4$. Proof of the theorem}

(4.1) Let $T$ be the 1-dimensional circle and let $t_{0}$ be a point of $T$. We denote by $T \vee T$ the subspace $\left(T \times t_{0}\right) \cup\left(T \times t_{0}\right)$ of the product space $T \times T$, and define mappings $u_{1}$ and $u_{2}:\left(T, t_{0}\right) \rightarrow\left(T \vee T, t_{0} \times t_{0}\right)$ by the formula

$$
u_{1}(x)=x \times t_{0}, \quad u_{2}(x)=t_{0} \times x, \quad(x \in T) .
$$

Let $\nu$ be a generator of $\pi_{1}\left(T, t_{0}\right)$ and let $\mu_{1}=u_{1 \pi}(\nu), \ell_{2}=u_{2 \pi}(\mu)$. Then it is well known that $\pi_{1}\left(T \vee T, t_{0} \times t_{0}\right)$ is a free group generated by $\mu_{1}$ and $\mu_{2}$. Since the canonical mapping $\omega_{1}: \pi_{1}\left(T \vee T, t_{0} \times t_{0}\right) \rightarrow \Pi_{1}\left(T \vee T, t_{0} \times t_{0}\right)$ is one-to-one, there exists a unique element $\kappa \in \pi_{1}\left(T \vee T, t_{0} \times t_{0}\right)$ such that

$$
\omega_{1}(\kappa)=\omega_{1}\left(\mu_{1}\right) \cdot \omega_{1}\left(\mu_{2}\right),
$$

and there exists a word $F(X, Y)$ in two variables $X$ and $Y$ such that

$$
\kappa=F\left(\mu_{1}, \mu_{2}\right) .
$$

Now let $\alpha$ and $\beta$ be two arbitrary elements in $\pi_{1}(A, a)$, and let $f$ and $g$ be con- 
tinuous mappings of $\left(T, t_{0}\right)$ to $(A, a)$ which represent the homotopy classes $\alpha$ and $\beta$ respectively. Let $f \vee g$ be the mapping of $\left(T \vee T, t_{0} \times t_{0}\right)$ to $(A, a)$ defined by the formula

$$
f \vee g\left(x \times t_{0}\right)=f(x), \quad f \vee g\left(t_{0} \times x\right)=g(x),
$$

Then

$$
\begin{aligned}
(f & \vee g)_{\mathrm{n}}\left(\omega\left(\mu_{1}\right) \cdot \omega\left(\mu_{2}\right)\right)=\left((f \vee g)_{\mathrm{n}} \omega\left(\mu_{1}\right)\right) \cdot\left((f \vee g)_{\mathrm{n}} \omega\left(\mu_{2}\right)\right) \\
& =\left(\omega\left\{(f \vee g)_{\pi} \mu_{1}\right\}\right) \cdot\left(\omega\left\{(f \vee g)_{\pi} \mu_{2}\right\}\right) \\
& =\omega(\alpha) \cdot \omega(\beta) .
\end{aligned}
$$

On the other hand, by (4.2) and (4.3),

$$
\begin{aligned}
(f & \vee g)_{\mathrm{n}}\left(\omega\left(\mu_{1}\right) \cdot \omega\left(\mu_{2}\right)\right)=(f \vee g)_{\mathrm{n}}\left(\omega\left(F\left(\mu_{1}, \mu_{2}\right)\right)\right. \\
& =\omega\left[(f \vee g)_{\pi}\left(F\left(\mu_{1}, \mu_{2}\right)\right)\right] \\
& \left.=\omega\left[F\left((f \vee g)_{\pi} \mu_{1},(f \vee g)_{\pi} \mu_{2}\right)\right)\right] \\
& =\omega(F(\alpha, \beta)) .
\end{aligned}
$$

Hence

$$
\omega(\alpha) \cdot \omega(\beta)=\omega(F(\alpha, \beta)), \quad \text { for every } \alpha, \beta \in \pi_{1}(A, a) .
$$

Here it must be remarked that the word $F(X, Y)$ does not depend on the pair ( $A, a)$.

Since $\omega_{1}$ is one-to-one and $\Pi_{1}\left(T \vee T, t_{0} \times t_{0}\right)$ is a group, we can define a group structure in the set $\pi_{1}\left(T \vee T, t_{0} \times t_{0}\right)$ by the formula

$$
\ddot{\alpha} \circ \beta=\omega^{-1}(\omega(\alpha) \cdot \omega(\beta)) \quad\left(\alpha, \beta \in \pi_{1}\left(T \vee T, t_{0} \times t_{0}\right)\right) \text {. }
$$

(4.4) implies that

$$
\alpha \circ \beta=F(\alpha, \beta) \quad\left(\alpha, \beta \in \pi_{1}\left(T \vee T, t_{0} \times t_{0}\right)\right) .
$$

Since $\pi_{1}\left(T \vee T, t_{0} \times t_{0}\right)$ is a free group generated by $\mu_{1}$ and $\mu_{2}$, by the lemma in $(2.9)$

$$
F(X, Y)=X Y, \text { or }=Y X .
$$

According to (4.4) and (4.5), we can easily verify that the canonical mapping $\omega_{1}$ is and isomorphism for each $(A, a)$, or an inverse-isomorphism for each $(A, a)$. As remarked in (3.3), this implies that $\omega_{n}: \pi_{n}(A, a) \rightarrow \Pi_{n}(A, a)$ is an isomorphism for each $(A, a)$ and $n \geq 0$, or an inverse-isomorphism for each 
$(A, a)$ and $n \geqslant 0$, and hence, by using the exact sequence (A.4), we can conclude that canonical mappings $\omega_{n}$ are all isomorphisms, or all inverse-isomorphisms.

(4.6) Now we have finished the proof of our Theorem, for we proved now the condition (1) in (1.7) and the condition (2) and (3) in (1.7) are proved in (3.4) and (3.2) respectively.

\section{REFERENCES}

[1] N. Iwahori and A. Hattori, On associative compositions in finite nilpotent groups, Nagoya Math. J., this number.

[2] A. Morimoto, A lemma on a free group, Nagoya Math. J., this number.

\section{Mathematical Institute,}

Nagoya University 